\title{
Developing Ideas and Methods for Supporting Whole Body Interaction in Remote Co-Design with Children
}

\author{
Minna O. Nygren* \\ UCL Knowledge Lab, University College London, minna.nygren@ucl.ac.uk \\ Marije Nouwen \\ Meaningful Interactions Lab (Mintlab), KU Leuven, marije.nouwen@kuleuven.be \\ Priscilla Van Even \\ Meaningful Interactions Lab (Mintlab), KU Leuven, priscilla.vaneven@kuleuven.be
}

Sara Price

UCL Knowledge Lab, University College London, sara.price@ucl.ac.uk

Bieke Zaman

Meaningful Interactions Lab (Mintlab), KU Leuven, bieke.zaman@kuleuven.be

Janne M. Beuthel

Center for HCI, University of Salzburg, jannemascha.beuthel@sbg.ac.at

Drawing from a present need as well as a growing interest towards developing methods to engage participants in research remotely [1,2], this workshop focuses on identifying opportunities and challenges around designing with children from an embodiment perspective $[\underline{3}, \underline{4}, \underline{5}, \underline{6}, \underline{10}, \underline{11}, \underline{12}, \underline{13}, \underline{15}]$. Themed around whole body interaction and digital technologies $[\underline{3}, \underline{7}, \underline{8}, \underline{9}, \underline{10}, \underline{11}, 14, \underline{15}, \underline{16}, \underline{17}, \underline{18}, \underline{20}, \underline{21}]$ the aim of this workshop is to gain methodological insights for the remote participation of children in design, and arrive at design insights addressing the question how we can design remote, inclusive whole body interactions for and with children while attending to child participants' multimodal communication and bodily engagement $[19,21]$ in a physically dislocated setting [1,2]. The workshop will involve discussions around data gathering from different angles, including relational, ethical, safety and practical perspectives, to create a comprehensive picture of what it entails to orchestrate embodied co-design workshops with children virtually. Outputs from the workshop will contribute to the IDC community by offering methodological insights from attendees' own experience and presented case studies from researchers and practitioners from both non-academic and academic settings to support children's embodied engagement in remote workshop situations and beyond.

CCS CONCEPTS • Human-centered computing Interaction design $\sim$ Interaction design process and methods $\sim$ Participatory design

Additional Keywords and Phrases: Whole Body Interaction Design, Co-Design with Children, Remote Connection, Technologies and Home

\section{ACM Reference Format:}

First Author's Name, Initials, and Last Name, Second Author's Name, Initials, and Last Name, and Third Author's Name, Initials, and Last Name. 2018. The Title of the Paper: ACM Conference Proceedings Manuscript Submission Template: This is the subtitle of the paper, this document both explains and embodies the submission format for authors using Word. In Woodstock'18: ACM Symposium on Neural Gaze Detection, June 03-05, 2018, Woodstock, NY. ACM, New York, NY, USA, 10 pages. NOTE: This block will be automatically generated when manuscripts are processed after acceptance.

\section{BACKGROUND}

Researchers and practitioners working with children have had to adapt to the variety of challenges brought by Covid-19 pandemic $[\underline{1}, \underline{22}]$. When face-to-face work has had to discontinue, new methodological challenges have emerged $[\underline{1}, \underline{2}, \underline{22}]$. Co-design is about communication and participation $[\underline{23}, \underline{24}]$ and work with whole body interaction requires the participation of a participant's body, therefore poses unique questions around eliciting, capturing, and enabling participation through whole body in a virtual setting.

Remote connection (i.e., connection over a space that is not physically shared), by physical or digital mediators, offers opportunities for participants over long distances within unprecedented, challenging circumstances. Participating in a remote research effort can have benefits, such as an ability to connect with new groups of participants, unexpected collaboration amongst researchers and practitioners synchronously during a research situation and utilizing new methods of communicating and participating. Participation always invites connection. However, remoteness also suggests disconnection. And adapting to a novel situation, such as remote research, inevitably changes dimensions of human connection and interaction. For example, for whole body interaction design in a remote setting, many

* Primary contact: minna.nygren@ucl.ac.uk 
challenges occur: issues relate to the body not being fully visible in a (web)camera to the present other; the technology may not be suitable enough to capture the whole body; and quality issues in internet connection may cause disruptions in the image of the moving body and limit access to what is present in the research situation. Other issues, such as engagement, time, place, and the presence of participants' caretakers are also factors contributing to the complexity of organizing such work.

It is against this background we would like to invite and engage participants to discuss and collaboratively think about ways in which to support multimodal and sensory engagement $[\underline{19}, \underline{21}, \underline{25}]$ in remote co-design events for whole body interaction with children. We are interested in participants bringing their own experience and questions to explore how we may leverage some of the existing methods in the area of whole body interaction design with children [ $\underline{5}, \underline{16}, 17]$, as well as embodied sketching [26] bodystorming [27], and somatoaesthetic approaches [25] to elicit ideas around whole body via, and despite, a remote connection; using a novel technology; or unexpected benefits of a larger international participant cohort. In so doing we aim to identify design opportunities and challenges around online co-design with children and whole body interaction and develop new ways of collaborating and investigating embodied design.

\section{WORKSHOP AIMS}

In this half-day workshop, we would like to create an interdisciplinary space that enables a group of academic and non-academic researchers and practitioners to come together and exchange and develop ideas and methods around 'remote design with and for children' (aged 2-18 years) from an embodiment perspective. Through presentations from invited guests, and exchanges of experiences from participants as well as a number of collaborative and participatory activities tailored for the workshop, our workshop aims to: (1) identify dimensions that support and hinder participatory interaction with and for children's whole body experiences and (2) define design strategies from an inclusion and participation perspective, and (3) identify and provide opportunities for innovative collaborations around whole body interaction design with children from an embodiment perspective.

\subsection{Workshop outputs}

- Collaborative journal article(s) drawing from ideas gathered during the workshop relating to design guidelines for remote whole body interaction design with children, specific focus left open to flexibly adjust the focus depending on the workshop outcomes

- List of key criteria/ guidelines for planning co-design workshops with children around whole body interaction design in remote settings which will be disseminated (please see page 5 "Post Workshop Plans" for details)

\section{ORGANIZERS}

The workshop organizers have been selected based on their diverse experience in embodied whole body interaction design with children in a variety of settings, and research methods development in remote interaction.

Minna Orvokki Nygren is a PhD Candidate at the UCL Knowledge Lab. Her doctoral work has investigated young children's affective engagement, body movement and embodied participation in meaning making as well as co-design of whole body interactive environments from an embodiment theory perspective to support embodied learning.

Marije Nouwen is a $\mathrm{PhD}$ Candidate at KU Leuven. In her $\mathrm{PhD}$ she focuses on how physically separated grandchildren and grandparents can communicate, care and play over distance with (tangible) technologies.

Priscilla van Even is a $\mathrm{PhD}$ candidate at KU Leuven. Her $\mathrm{PhD}$ focuses on rethinking science communication and uses participatory methods to develop a framework for meaningful and engaging science dissemination. In the past, she has worked on a project that focuses on participatory design in art and design museums with children

Sara Price is Professor of Digital Learning at UCL Knowledge Lab. Her work focuses on design and evaluation of emergent digital technologies for learning and developing methodological approaches for researching embodiment.

Bieke Zaman is Associate Professor in Human-Computer Interaction and research group leader of the Meaningful Interactions Lab (Mintlab), part of the Institute for Media Studies at the Faculty of Social Sciences, KU Leuven, Belgium. She has substantial experience in the field of IDC where her research programmes encompass children and technology, media convergence, and progressive methods including creative forms of research outreach.

Janne Mascha Beuthel is a PhD student at the Center for Human-Computer Interaction at the University of Salzburg. In her research, she combines practices from textile design, principles from participatory design with wearable technologies to speculate on better inclusion and understanding of diverse bodily experiences.

\section{WEBSITE}

A website for this workshop has been set up at www.remotewholebodyinteraction.wordpress.com. This website will function as a space for organisers to centralise useful information for workshop participants, gather resources relating to the workshop themes, and postworkshop it will also be used to disseminate findings and future work that draws from the workshop. 


\section{PRE-WORKSHOP PLANS}

A call for participation will be shared through IDC, across social media, relevant mailing lists (e.g., CHI, TEI) and personal communication channels. We will also announce the workshop on the websites of organisers' institutions. This workshop partly spawns from a recent project on designing embodied and sensorimotor interaction for science learning in informal learning contexts [28]. We will also reach out to researchers in related fields as well as key non-academic communities including game design. Therefore, we estimate we will be able to attract a diverse community of minimum 12-15 participants.

Submissions will be reviewed by the organisers and invited reviewers using criteria described in the Call for Participation. Following the alt-chi model, reviewers and articles are not anonymized. Authors will receive notification by the end of May and will be asked to register for the workshop.

\section{WORKSHOP STRUCTURE}

A half day workshop with participants is planned to take place virtually on 27 June 2021 13:00-16:45 (GMT+1) during IDC 2021 Conference. Workshop activities and discussions will take place both within the whole group and in smaller groups. Organisers will draw specific ideas, questions, and concerns from participants' position papers that will shape workshop activities and themed discussions. Participants' position papers will also shape post workshop plans, which will be identified pre-workshop to give participants the opportunity to identify how they would like to contribute to taking learnings from the workshop forwards (e.g. working groups with specific teams). Position papers will also inform organisers about participants' technical tool preferences (e.g. Miro, Teams, Zoom) which will help support accessibility.

\subsection{Schedule}

13:00 Introductions from everyone, and goals of the workshop ( $25 \mathrm{~min})$

13:25 Presentation from Marije Nouwen (Mintlab, KU Leuven) on the use of remote probes in interaction research with children (6-10 years) and their grandparents to explore dislocated and asynchronous play environments (5 min)

13:30 Presentation from Lili Golmohammadi (UCL Knowledge Lab) on remote participatory workshops with international research participants during pandemic $(5 \mathrm{~min})$

13:35 Design contexts: experiences from the field. Whole group discussion to share learnings. Specific common themes conveyed by participants are identified. (45 min)

14:05 Break (15 min), option to stay and chat or get up and stretch.

14:20 Small group discussions based on learnings from the first group activity to identify opportunities, challenges, and solutions/guidelines in relation to design contexts. (45 min)

15:05 Break (10 min), option to stay and chat or get up and stretch.

15:15 Presentations of small group work from the previous activity. Whole group discussion around gathered examples and identifying key design guidelines as proposed solutions to that enables children's whole body interaction in remote co-design settings. (45min)

16:00 Whole group plenary activity. (15 mins)

16:15-16:30 Workshop end, thank yous and well wishes before we say goodbye.

\section{POST WORKSHOP PLANS}

The format of the outcomes after the workshop will be shaped by (1) the prospective participants' submissions and a pre-workshop thought gathering, in which we ask about the types of collaborative activities they would like to become involved in, and (2) findings that emerge during the workshop based on participants' discussions around the workshop themes. We ask for successful applicants to fill in a sign-up sheet for post-workshop working groups for example for co-writing and article with design guidelines drawn from the ideas from the workshop, research proposal planning or other activity. Specific meeting dates for each working group will be proposed to ensure the workshop activities will continue.

Possible publication outlets include conferences (e.g. CHI) or relevant journals, and publications will also be shared on the workshop webpage and circulated across the IDC community.

Furthermore, we will publish a summary of insights from the workshop on the workshop website www.remotewholebodyinteraction.wordpress.com as well as a dissemination blog post distributed via organisers' institutions and social media.

\section{CALL FOR PARTICIPATION}

\subsection{Title}

Developing Ideas and Methods for Supporting Whole Body Interaction in Remote Co-Design with Children 


\subsection{The format and goals of the workshop}

Half Day virtual workshop (see Workshop Structure for more details). The goals of the workshop are:

- Gather examples of work to date around remote embodied design with children and young people aged 2-18 years

- Identify dimensions of practical and theoretical approaches that support remote design for and with children from an embodiment perspective

- Development of practical tools, technologies, and ideas to support children's participation and inclusion in remote settings.

- Launch collaboration opportunities that take learnings from the workshop further after the workshop through specific post-workshop working groups (for example to co-write an article, plan research proposal or other, suggested by participants in their position paper submission and crystallised via pre-workshop thought gathering)

\subsection{Submission}

Up to 2-page submission paper using CHI Extended Abstracts format: https://www.acm.org/publications/taps/word-template-workflow

\subsection{Requirements for position papers}

We invite submissions from academic and non-academic practitioners with research or practice-based experience with children and young people (aged 2-18 years) and who have worked or have an interest in working with children in a remote setting from a whole body perspective. The workshop's main focus is understanding ways in which to support of whole body interaction and multimodal communication. We are interested in developing methods and ideas around conducting such work through body-based activity and understanding design implications, for example, in designing tangible, movement-based or virtual interaction experiences for and with children.

In their submission, prospective participants are asked to:

- $\quad$ outline their research or practice-based work experience with children and digital technologies

- identify challenges and strategies for co-design methods development in a remote setting when the focus is on preserving the notion of the whole body in interaction

- $\quad$ reflect on whole body perspective of involving children in research. This could be, for example, a position statement from the perspective of critical design, or a set of research questions.

- A personal aspiration should revolve around the question on how to be inclusive when doing remote co-design with children, and whether our current, new and adopted theoretical frameworks open-up our analytical lens when performing and analysing whole body interactions in remote-research settings.

\subsection{The participant selection criteria}

Workshop organisers review submissions from prospective participants according to the ways in which these can invoke inspirational and even surprising ideas around the themes of the workshop, as well as the diversity of background and experiences.

\section{ACKNOWLEDGEMENTS}

This material is based upon work supported under a collaboration between the National Science Foundation (NSF), the Wellcome Trust, and the Economic and Social Research Council (ESRC) via a grant from the NSH (NSF grant no. 1646940) and a grant from the Wellcome Trust with ESRC (Wellcome Trust grant no. 206205/Z/17/Z). Any opinions, findings and conclusions or recommendations expressed in this materials are those of the authors and do not necessarily reflect the view of NSF, the Wellcome Trust, or ESRC. In addition, we gratefully acknowledge the financial support by the Flemish Fund for Scientific Research (FWO - grant number G0E3718N), European Union's Horizon 2020 research and innovation programme under grant agreement No 872500, and the Austrian Science Fund (FWF): I $3580-N 33$.

\section{REFERENCES}

$<$ bib id="bib1" $><$ number $>[1]<$ number $>$ Kung Jin Lee, Wendy Roldan, Tian Qi Zhu, Harkiran Kaur Saluja, Sungmin Na, Britnie Chin, Yilin Zenf, Jin Ha Lee, Jason Yip. 2020. The Show Must Go On: A conceptual model of conducting synchronoys participatory design with children online. Submitted to Proceedings of the $2021 \mathrm{CHI} C$ onference on Human Factors in Computing Systems. Association for Computing Machinery. [PDF-preprint] $</$ bib $>$

$<$ bib id="bib2" $><$ number $>[2]<$ number $>$ Verena Fuchsberger, Janne Mascha Beuthel, Dorothé Smit, Philippe Bentegeac, Manfred Tscheligi, Marije Nouwen, Bieke Zaman, Tanja

Döring. Designing for Tangible (Un-) Connectedness 2020. In DIS '20 Companion, July 6-10, 2020, Eindhoven, Netherlands. DOI: https://doi.org/10.1145/3393914.3395916 $<$ bib $>$

$<$ bib id="bib3" $><$ number $>[3]</$ number $>$ Nikoleta Yiannoutsou, R. Johnson \& Sara Price. (accepted) Non-visual Virtual Reality: Considerations for the Pedagogical Design of Embodied Mathematical Experiences for Visually Impaired Children. To be published in the Special Issue on 'Learning Experience Design: Embodiment, Gesture, and Interactivity in XR' in Education, Technology \& Society $</$ bib $>$

$<$ bib id="bib4" $><$ number $>[4]<$ number $>$ Rhiannon Thomas Jha, Sara Price and Alison Motion. 2020. Designing 'Embodied' Science Learning Experiences for Young Children. Lecture Notes in Computer Science 12205, Learning and Collaboration Technologies. Designing, Developing and Deploying Learning Experiences. 7th Internationa Conference, LCT 2020, held as Part of the 22nd HCI International Conference, HCII 2020, Copenhagen, Denmark, July 19-24, 2020, Proceedings, Part I, pp. 207-225</bib> $<$ bib id="bib5" $><$ number $>[5]<$ /number $>$ Alissa N. Antle, Paul Marshall and Elise van den Hoven (Eds.). 2011. Workshop Proceedings. Embodied Interaction: Theory and Practice in HCI. Workshop at CHI 2011, May 7-12, Vancouver BC, Canada. $<$ bib $>$

$<$ bib id="bib6" $><$ number $>[6]<$ /number $>$ Dor Abrahamson, Carmen J. Petrick, H. Taylor Martin, David J. DeLiema, Noel Enyedy, Francis F. Sten, Darin Hoyer, Mina C. JohnsonGlenberg, David Birchfield, Tatyana Koziupa, Caroline Savio-Ramos, Julie Cruse, Robb Lindgren, Anthony Aakre, J. Michael Moshell, Cameron L. Fadjo, John B. Black, and Mike Eisenberg. 2012. You're it! Body, action, and object in STEM learning. In J. van Aalst, K. Thompson, M. J. Jacobson, P. Reimann (Eds.) Proceedings of the 10th International Conference of the Learning Sciences (ICLS '12), 99-109.</bib > 
$<$ bib id="bib7" $><$ number $>[7]</$ number $>$ Ulrike Cress, Ursula Fischer, Korbinian Moeller, Claudia Sauter, Hans-Christoph Nuerk. 2010. The use of a digital dance mat for training kindergarten children in a magnitude comparison task. In Proceedings of the 9th international Conference of the Learning Sciences (ICLS '10), 105-112.</bib $>$

$<$ bib id="bib8" $><$ number $>[8]<$ number $>$ Kelly Yap, Clement Zheng, Angela Tay, Ching- Chiuan Yen, and Ellen Yi-Luen Do. 2015. Word Out!: learning the alphabet through full body interactions. In Proceedings of the 6th Augmented Human Interactional Conference (AH '15), 101-8. https://doi.org/10.1145/2735711.2735789 </bib $>$

$<$ bib id="bib9" $><$ number $>[9]</$ number $>$ Darren Edge, Kai-yin Cheng, and Michael Whitney. 2013. SpatialEase: Learning Language through Body Motion. In Proceedings of the SIGCHI Conference on Human Factors in Computing Systems (CHI '13), 469-472. https://doi.org/10.1145/2470654.2470721</bib $>$

$<$ bib id="bib10" $><$ number $>[10]<$ number $>$ Robb Lindgren, Michael Tscholl, and J. Michael Moshell. 2014. MEteor: Developing Physics Concepts Through Body-Based Interaction with a Mixed Reality Simulation. Physics Education Research Conference (PERC '13), 217-220. https://doi.org/10.1119/perc.2013.pr.042 </bib $>$

$<$ bib id="bib11" $><$ number $>[11]<$ /number $>$ Noel Enyedy, Joshua A. Danish, Girlie Delacruz, and Melissa Kumar. 2012. Learning physics through play in an augmented reality environment. International Journal of Computer- Supported Collaborative Learning, 7(3): 347-378. https://doi.org/10.1007/s11412-012-9150-3</bib>

$<$ bib id="bib12" $><$ number $>[12]</$ number $>$ Dor Abrahamson and Robb Lindgren. 2014. Embodiment and Embodied Design. In The Cambridge Handbook of the Learning Sciences (2nd. ed.), R. Keith Sawyer (Ed.). Cambridge University Press, Cambridge, UK, 358-76. https://doi.org/10.1017/CBO9781139519526.022 </bib> $<$ bib id="bib13" $><$ number $>[13]</$ number $>$ Mina C. Johnson-Glenberg. Immersive VR and Education: Embodied Design Principles That Include Gesture and Hand Controls. Frontiers in Robotics and AI 5: 27-19. https://doi.org/10.3389/frobt.2018.00081</bib $>$

$<$ bib id="bib14" $><$ number $>[14]</$ number $>$ Jason Mickelson and Wendy Ju. 2011. Math Propulsion: Engaging Math Learners Through Embodied Performance \& Visualization. In Proceedings of the fifth international conference on Tangible, embedded, and embodied interaction (TEI'11), 101-108. https://doi.org/10.1145/1935701.1935722 </bib $>$

$<$ bib id="bib15" $><$ number $>[15]</$ number $>$ Minna Orvokki Nygren and Sara Price. 2020. EPESonic: Understanding fictional framing of metaphorical actions in young children's digital interaction. Proceedings of International Conference on Interaction Design and Children, June 2020, London $</$ bib $>$

$<$ bib id="bib16" $><$ number $>[16]<$ number $>$ Marie-Monique Schaper, Ole Sejer Iversen, Laura Malinverni and Narcis Pares. 2019. FUBImethod: Strategies to engage children in the co-design of Full-Body interactive experiences. In International Journal of Human-Computer Studies (132): 52-69. DOI: https://doi.org/10.1016/j.ijhcs.2019.07.008 </bib >

$<$ bib id="bib17" $><$ number $>[17]</$ number $>$ Franscesco Cafaro, Leilah Lyons and Alissa N. Antle. 2018. Framed Guessability: Improving the Discoverability of Gestures and Body Movements for Full-Body Interaction. CHI 2018; April 21-26,2018, Montreal, QC, Canada. DOI: https://doi.org/10.1145/3173574.3174167 </bib>

$<$ bib id="bib18" $><$ number $>[18]</$ number $>$ Seokbin Kang, Leyla Norooz, Vanessa Oguamanam, Angelisa C. Plane, Tamara L. Clegg, Jon E. Froelich. 2016. SharedPhys: Live physiological sensing, whole-body interaction, and large-screen visualizations to support shared inquiry experiences. In IDC-16, June 21-24, 2016, Manchester, England, UK. DOI: http://dx.doi.org/10.1145/2930674.2930710 $</$ bib $>$

$<$ bib id="bib19" $><$ number $>[19]</$ number $>$ Carey Jewitt, Anna Xambo and Sara Price. "Exploring methodological innovation in the social sciences: the body in digital environments and the arts." In International Journal of Social Research Methodology, 20(2017): 10-5-120. DOI: http://dx.doi.org/10.1080/13645579.2015.1129143 </bib> $<$ bib id="bib20" $><$ number $>[20]</$ number $>$ David England (Ed.). 2011. Whole Body Interaction. London: Springer. $<$ /bib $>$

$<$ bib id="bib21" $><$ number $>[21]</$ number $>$ Sara Price, Mona Sakr and Carey Jewitt. 2016. Exploring Whole-Body Interaction and Design for Museums. In Interacting with Computers, 28, 5 (2016), 569-583.</bib $>$

$<$ bib id="bib22" $><$ number $>[22]<$ /number $>$ Natalia Kucirkova, Cecilie Evertsen-Stanghelle, Ingunn Sudsrød, Ida Bruheim Jensen, Ingunn Størksen. 2020. Lessons for childcomputer interaction studies following the research challenges during the Covid-19 pandemic. In International Journal of Child-Computer Interaction, 26 (2020), 1-7. https://doi.org/10.1016/j.ijcci.2020.100203 $<$ /bib $>$

$<$ bib id="bib23" $><$ number $>[23]</$ number $>$ Alison Druin. 2002. "The role of children in the design of new technology." In Behaviour \& Information Technology, 21, 1(2002), 125. https://doi.org/10.1080/01449290110108659</bib $>$

$<$ bib id="bib24" $><$ number $>[24]<$ number $>$ Selina Schepers, Katrien Dreessen and Bieke Zaman. 2018. Rethinking children's roles in Participatory Design: The child as a process designer. In International Journal of Child-Computer Interaction. $16(2018) 47-54 .</$ bib $>$

$<$ bib id="bib25" $><$ number $>[25]</$ number $>$ Kristina Höök. 2018. Designing with the Body: Somatoaesthetic Design. Cambridge, MA: The MIT Press. $<$ /bib $>$

$<$ bib id="bib26" $><$ number $>[26]<$ /number $>$ Elena Márquez Segura, Laia Turmo Vidal, Asreen Rostami and Annika Waern. 2016. Embodied Sketching. CHI'16, San Jose, CA, USA. http://dx.doi.org/10.1145/2858036.2858486 </bib $>$

$<$ bib id="bib27" $><$ number $>[27]<$ number $>$ Antti Oulasvirta, Esko Kurvinen, and Tomi Kankainen. 2003. Understanding Contexts by Being There: Case Studies in Bodystorming. Personal Ubiquitous Comput. 7, 2 (2003), 125-134. http://doi.org/10.1007/s00779-003-0238-7 </bib >

$<$ bib id="bib28" $><$ number $>[28]<$ /number $>$ Move2Learn. 2017-2021. Embodied Science Learning for Children in Museums. Retrieved February 14, 2021 from www.move2learn.net $</$ bib $>$ 